\title{
Sucesso do Outubro Rosa no Brasil: uma boa notícia para o controle do câncer de mama no país?
}

Arn Migowski 1,2

doi: 10.1590/0102-311X00247121

Em artigo publicado nesta edição, Baquero et al. 1 avaliaram a tendência temporal e a sazonalidade das buscas dos termos "câncer de mama" e "mamografia" no Google Trends entre 2004 e 2019, bem como sua correlação com exames mamográficos no Sistema Único de Saúde (SUS). Os dados apresentados demonstram claro padrão sazonal com picos de ambos termos de busca em outubro, o que, por sua temporalidade, sugere fortemente uma relação causal com a campanha Outubro Rosa. Os resultados mostram ainda distribuição espacial semelhante entre os dois termos, com forte correlação, bem como um aumento da sazonalidade nos anos mais recentes. Outros estudos usando Google Trends, para avaliar o interesse em rastreamento de câncer em outros países, têm sido realizados nos últimos anos e também têm demonstrado o mesmo padrão de sazonalidade encontrado no estudo brasileiro 2,3. São evidências de um indubitável sucesso do Outubro Rosa. Contudo, o que poderia ser uma boa notícia, em se tratando de uma campanha de saúde pública, na verdade, traz um cenário complexo e preocupante.

Como no Brasil o rastreamento é oportunístico, sem convite individualizado para a população-alvo, há um importante papel de campanhas midiáticas no sentido de mobilizar as mulheres ${ }^{4}$. Nesse sentido, o maior benefício da campanha Outubro Rosa, no que tange aos exames de rotina, seria contribuir para o aumento da cobertura do rastreamento bienal na faixa etária de 50 a 69 anos 5 . Os dados da nova Pesquisa Nacional de Saúde, divulgados em setembro de 2021, indicam evolução da cobertura em 2019, especialmente nas regiões com resultados piores em 2013, e indicam redução da proporção de mulheres na população-alvo que nunca realizaram o exame, com relação a 2013 (24,2\% vs. 31,5\%) 6. Como na recomendação atual de periodicidade - dois anos é o intervalo mínimo - a cobertura seria maior do que com a pergunta do inquérito ${ }^{4}$. Por outro lado, por ser uma informação autorreferida, pode estar superestimada em virtude de viés do respondente. De qualquer forma, são dados mais representativos do que os do inquérito telefônico Vigilância de Fatores de Risco e Proteção para Doenças Crônicas por Inquérito Telefônico (Vigitel) ou do Sistema de Informação do Câncer 7 . Embora a campanha deva ter alguma influência positiva nos resultados de cobertura, são muitos os motivos de preocupação com o conteúdo que vem sendo predominantemente veiculado no Brasil ao longo dos anos.
1 Instituto Nacional de Câncer José Alencar Gomes da Silva, Rio de Janeiro, Brasil.

2 Coordenação de Ensino e Pesquisa, Instituto Nacional de Cardiologia, Rio de Janeiro, Brasil. 
Inferências equivocadas a partir de uso de desfechos como risco relativo e de sobrevida, superdimensionam os benefícios do rastreamento e têm sido usadas exaustivamente nas campanhas 8. Há ainda uma centralidade na ideia de que o tamanho do tumor é o único fator prognóstico, hipótese baseada no paradigma Halstediano, desenvolvido no século XIX. Essa teoria baseia-se no conceito de que um caso de câncer se origina de um único local, cresce nesse local e com o tempo migra para linfonodos, e depois se dissemina para órgãos distantes. Ela foi de grande importância para o entendimento da história natural do câncer e é uma das bases da classificação de estadiamento de câncer. Contudo, ela não é capaz de explicar toda a heterogeneidade do comportamento biológico do câncer de mama ou fenômenos como micrometástases ou sobrediagnóstico e suas hipóteses não se verificaram nem no seguimento dos ensaios clínicos randomizados de rastreamento mamográfico, nem em estudos observacionais nos países com ampla difusão desse rastreamento 9 .

O rastreamento mamográfico fora da faixa etária de 50 e 69 anos e em periodicidade menor do que bienal está associado a expressivo aumento de riscos, sem comprovação conclusiva de benefício adicional 5 . Todavia, o conteúdo das mensagens da campanha não tem contribuído para adesão a essas diretrizes. Geralmente voltada para mulheres jovens, a campanha Outubro Rosa claramente é um vetor no sentido de indução da realização de rastreamento fora da população-alvo. A característica anual da campanha reforça ainda mais a prática de periodicidades menores do que a recomendada 4 . Em estudo que avaliou o conteúdo de matérias jornalísticas publicadas no Brasil em outubro, o rastreamento mamográfico apareceu em cerca de $80 \%$ das matérias, mas predominou a recomendação de iniciá-lo aos 40 anos 10. Esse estudo mostrou ainda que a recomendação oficial de rastreamento bienal de 50 a 69 anos foi citada em apenas 17,5\% das matérias; apenas 3,3\% citaram os riscos do rastreamento; $1,5 \%$, a importância da decisão compartilhada e apenas $11,8 \%$ listaram todas os principais sinais e sintomas suspeitos de câncer de mama 10. No exterior, há mais de uma década, já há forte movimento para mudança desse tipo de comunicação enviesada, mas no Brasil, ele ainda é incipiente e contra-hegemônico 4,10.

As inadequações da prática de rastreamento no SUS são ainda maiores do que as mostradas por Baquero et al. 1. Mesmo o rastreamento considerado como "em conformidade" pelos autores, não considera a questão da periodicidade, por limitação dos indicadores utilizados. Ademais, a antiga recomendação de antecipação de rastreamento mamográfico para população de alto risco não está mais vigente desde 2015 5. O resultado é um padrão de rastreamento com baixa eficiência e eficácia, que traz diversos riscos desnecessários às mulheres. Variados fatores além da própria campanha explicam essas inadequações do rastreamento no Brasil, tais como a medicina defensiva 4 e a existência de diretrizes com menor rigor metodológico, baseadas em consenso de especialistas 8 . Esses dois fatores são reforçados pelo fato de os principais riscos do rastreamento serem percebidos paradoxalmente pelas mulheres e médicos assistentes como benefícios ${ }^{4}$. A tendência temporal do número de mamografias no SUS por faixa etária apresentada pelos autores ${ }^{1}$ possui outros determinantes ligados às novas diretrizes de detecção precoce, forma de financiamento, entre outros, e que estão em constante tensão ${ }^{4}$. Embora os dados apresentados sejam muito representativos 1, também podem ter interferido na série temporal o uso de motores de busca como Yahoo e Bing, ou mudanças no padrão de busca a partir da última década, com crescente procura por vídeos ou até mesmo uso de redes sociais como Facebook, Instagram ou Twitter para fins de busca. Independente disso, os resultados apresentados por Baquero et al. 1, apontam para um reducionismo ainda maior na campanha brasileira do que nos 
Estados Unidos, com maior foco em torno do exame de mamografia. Mas o que poderia substituir a mamografia como principal mote da campanha?

Apesar de Baquero et al. 1 citarem métodos de rastreamento que poderiam ser mais promissores, ainda não há evidências de que outro exame poderia substituir a mamografia. $\mathrm{O}$ exame clínico das mamas foi historicamente incentivado no Brasil como alternativa ao rastreamento mamográfico, em especial em mulheres jovens, em função dos diversos problemas do rastreamento mamográfico nesse grupo. Mas até ele está com esse papel em cheque. Ele havia sido colocado em dúvida nas diretrizes atuais, na expectativa da publicação de resultados de ensaios clínicos randomizados 4,5. Em 2021, foram publicados os resultados de 20 anos de seguimento do ensaio clínico randomizado de rastreamento com exame clínico das mamas bienal, em Mumbai, na Índia, mas não foi possível observar redução estatisticamente significativa da mortalidade 11. Com base numa análise post hoc de subgrupo os autores alegam que apenas em mulheres com mais de 50 anos ele parece eficaz, mas ainda que fôssemos considerar esses resultados, eles dizem respeito ao exame clínico das mamas como método de rastreamento isolado e não em paralelo à mamografia 11.

Em vez de tentar substituir o método de rastreamento, o ideal para o contexto brasileiro seria o fortalecimento das estratégias de diagnóstico precoce, voltadas a agilizar a investigação diagnóstica em mulheres com sinais e sintomas iniciais da doença ${ }^{12}$. Nas diretrizes para detecção precoce do câncer de mama no Brasil foram recomendadas três estratégias de diagnóstico precoce: estratégia de conscientização, protocolo de encaminhamento prioritário de casos com sinais e sintomas suspeitos e a confirmação diagnóstica em um único serviço ${ }^{5}$. Essas estratégias dependem fortemente da organização do sistema de saúde, regulação assistencial, planejamento de rede e logística para otimização de processos 4 . É por isso que países como a Dinamarca conseguiram redução do tamanho médio dos tumores de mama em 10 anos $(9 \mathrm{~mm})$ maior do que nos ensaios de rastreamento mamográfico 13. Isso é reforçado pela redução da letalidade dos cânceres palpáveis nas últimas décadas por melhorias no tratamento do câncer de mama, diminuindo a efetividade relativa do rastreamento 14 . Porém, essas estratégias têm menor apelo midiático, e certamente é um enorme desafio conseguir pautá-las para a mídia de massa, gestores, legisladores, advocacy e população em geral. Isso se reflete não apenas nas campanhas, mas até mesmo nas pesquisas, com pouca atenção dada ao diagnóstico precoce, criando um ciclo vicioso de baixa geração de evidências 4,5 .

A pandemia de COVID-19 não só reduziu o rastreamento de câncer em todo o mundo por múltiplos determinantes, mas também diminuiu em até $76 \%$ o volume de buscas no Google pelo tema, com uma redução mais acentuada do que nas buscas por doenças crônicas em geral 15. Com o progressivo retorno ao interesse no Outubro Rosa, há a oportunidade de tentar ressignificar a campanha, voltando a mesma para estratégias mais efetivas, a fim de reduzir o inevitável impacto do contexto pandêmico no atraso no diagnóstico de câncer.

Nos moldes atuais, provavelmente, o sucesso do Outubro Rosa no Brasil traz mais danos do que benefícios para a saúde das mulheres. Ainda assim, o grande interesse despertado pela campanha, bem demonstrado por Baquero et al. 1, e o crescente acesso de população com idades mais avançadas à Internet são janelas de oportunidade para que sejam pautadas informações de qualidade e relevância que possam superar o paradigma reducionista e equivocado que está posto, com disseminação de informações corretas que permitam empoderamento das mulheres na tomada de decisão compartilhada sobre o rastreamento, mas 
também a tão necessária mudança de foco para as ações de diagnóstico precoce. Lembrando sempre que essas ações começam na estratégia de conscientização das mulheres, mas precisam ter uma correspondência no fortalecimento do SUS.

\section{Informação adicional}

ORCID: Arn Migowski (0000-0002-4861-2319).
1. Baquero OS, Rebolledo EAS, Ribeiro AG, Bermudi PMM, Pellini ACG, Failla MA, et al. Outubro Rosa e mamografias: quando a comunicação em saúde erra o alvo. Cad Saúde Pública 2021; 37:e00149620.

2. Schootman M, Toor A, Cavazos-Rehg P, Jeffe DB, McQueen A, Eberth J, et al. The utility of Google Trends data to examine interest in cancer screening. BMJ Open 2015; 5:e006678.

3. Mohamad M, Kok HS. Using Google Trends data to study public interest in breast cancer screening in Malaysia. Asian Pac J Cancer Prev 2019; 20:1427-32.

4. Migowski A, Dias MBK, Nadanovsky P, Azevedo e Silva G, Sant'Ana DR, Stein AT. Diretrizes para detecção precoce do câncer de mama no Brasil. III - Desafios à implementação. Cad Saúde Pública 2018; 34:e00046317.

5. Migowski A, Azevedo e Silva G, Dias MBK, Diz MDPE, Sant'Ana DR, Nadanovsky P. Diretrizes para detecção precoce do câncer de mama no Brasil. II - Novas recomendações nacionais, principais evidências e controvérsias. Cad Saúde Pública 2018; 34:e00074817.

6. Instituto Nacional de Câncer José Alencar Gomes da Silva. Dados e números sobre câncer de mama. Rio de Janeiro: Instituto Nacional de Câncer José Alencar Gomes da Silva; 2021. 
7. Instituto Nacional de Câncer José Alencar Gomes da Silva. Informativo: detecção precoce. Boletim 2020; 11(1). https://www.inca.gov. $\mathrm{br} / \mathrm{sites} / \mathrm{ufu}$.sti.inca.local/files//media/docu ment//informativo_numero1_2020.pdf (acessado em 15/Out/2021).

8. Migowski A, Stein AT, Ferreira CBT, Ferreira DMTP, Nadanovsky P. Diretrizes para detecção precoce do câncer de mama no Brasil. I - Métodos de elaboração. Cad Saúde Pública 2018; 34:e00116317.

9. Welch HG, Gorski DH, Albertsen PC. Trends in metastatic breast and prostate cancer: lessons in cancer dynamics. N Engl J Med 2015; 373:1685-7.

10. Assis M, Santos ROM, Migowski A. Detecção precoce do câncer de mama na mídia brasileira no Outubro Rosa. Physis (Rio J.) 2020; 30:e300119.

11. Mittra I, Mishra GA, Dikshit RP, Gupta S, Kulkarni VY, Shaikh HKA, et al. Effect of screening by clinical breast examination on breast cancer incidence and mortality after 20 years: prospective, cluster randomised controlled trial in Mumbai. BMJ 2021; 372:n256.
12. dos-Santos-Silva I. Políticas de controle do câncer de mama no Brasil: quais são os próximos passos? Cad Saúde Pública 2018; 34:e00097018.

13. Jørgensen KJ, Gøtzsche PC, Kalager M, Zahl PH. Breast cancer dcreening in Denmark: a cohort study of tumor size and overdiagnosis. Ann Intern Med 2017; 166:313-23.

14. Welch HG, Prorok PC, O'Malley AJ, Kramer BS. Breast-cancer tumor size, overdiagnosis, and mammography screening effectiveness. $\mathrm{N}$ Engl J Med 2016; 375:1438-47.

15. Snyder A, Jang S, Nazari IS, Som A, Flores EJ, Succi MD, et al. Google search volume trends for cancer screening terms during the COVID-19 pandemic. J Med Screen 2021; 28:210-2. 